\title{
Optimized Low Dispersion and Low Dissipation Runge- Kutta Algorithms in Computational Aeroacoustics
}

\author{
Appanah Rao Appadu* \\ Department of Mathematics and Applied Mathematics, University of Pretoria, Pretoria 0002, South Africa
}

Received: 7 Mar. 2013, Revised: 8 Jul. 2013, Accepted: 9 Jul. 2013

Published online: 1 Jan. 2014

\begin{abstract}
A new explicit fourth-order six-stage Runge-Kutta scheme with low dispersion and low dissipation properties is developed. This new Runge-Kutta scheme is shown to be more efficient in terms of dispersion and dissipation properties than existing algorithms such as Runge-Kutta temporal schemes developed by Hu et al. (1996), Mead and Renaut (1999), Tselios and Simos (2005). We perform a spectral analysis of the dispersion and dissipation errors. Numerical experiments dealing with wave propagation are performed with these four temporal Runge-Kutta schemes, all coupled with a nine-point centred difference scheme of eight order. The variation of two types of error: the error rate with respect to the $L_{1}$ norm and the Total Mean Square Error, both with respect to the CFL are obtained for all the four different schemes and it is seen that low CFL numbers are in general more effective than larger CFL numbers.
\end{abstract}

Keywords: Runge-Kutta, dispersion, dissipation, CFL

\section{Introduction}

In Computational Aeroacoustics (CAA), the accurate prediction of the generation of sound is demanding due to the requirement for preservation of the shape and frequency of wave propagation and generation. It is well-known $[11,23]$ that in order to conduct satisfactory computational aeroacoustics, numerical methods must generate the least possible dispersion and dissipation errors. In general, higher order schemes would be more suitable for CAA than the lower-order schemes since, overall, the former are less dissipative [13]. This is the reason why higher-order spatial discretisation schemes have gained considerable interest in computational aeroacoustics.

Since multidimensional finite-volume algorithms are generally more expensive in terms of numerical cost than finite-difference algorithms, the majority of CAA codes are based on the finite-difference methodology [8]. The trend within the field of CAA has been to employ higher-order accurate numerical schemes that have in some manner been optimized for wave propagation in order to reduce the number of grid points per wavelength while ensuring tolerable levels of numerical error. We now highlight some developments made in the construction of low dispersion and low dissipation Runge-Kutta algorithms.

$\mathrm{Hu}$ et al. [14] have constructed Runge-Kutta methods with low dispersion and low dissipation properties. They minimize the error between the exact and the numerical solution by determining the required coefficients. Stanescu and Habashi [21] propose a special Runge-Kutta scheme that can be written using minimum storage (i.e $2 \mathrm{~N}$-storage where $\mathrm{N}$ is the dimension of the first order differential system). Mead and Renaut [19] propose a six-stage fourth order Runge-Kutta method, in the context of Chebyshev pseudospectral discretization, with extended stability along the imaginary axis. Berland et al. [9] have constructed an explicit low-storage fourth-order six-stage Runge-Kutta scheme optimized in the Fourier space with a large stability range.

\section{Organisation of paper}

In section 3, we obtain estimates for the dispersion and dissipation errors in a six-stage fourth order Runge-Kutta scheme containing two parameters, $\beta_{5}$ and $\beta_{6}$. Section 4 highlights the salient features of some of our techniques

\footnotetext{
*Corresponding author e-mail: Rao.Appadu@up.ac.za, biprao2@yahoo.com
} 
of optimisation. In section 5, we use our techniques of optimisation to find optimal values of $\beta_{5}$ and $\beta_{6}$ and then perform a comparison of the spectral analysis of the dispersive and dissipative properties of the Runge Kutta schemes constructed by Hu et al. [14], Mead and Renaut [19], Tselios and Simos [25] with our new Runge-Kutta scheme. In section 6 , we present the results of a numerical experiment dealing with a convective wave equation and a non-linear spherical wave problem using four different schemes and compare some types of errors. Section 7 highlights the main results of this paper.

\section{Dispersion and Dissipation in Runge-Kutta Methods}

We consider the time integration using Runge-Kutta algorithms of the differential equation

$$
\frac{\partial u}{\partial t}=F(u, t),
$$

where the operator, $F$ is a function of the unknown, $u$ and of time, $t$.

$\mathrm{Hu}$ et al. [14] proposed the following low-storage $s$-stage algorithm to compute the time integration from $u^{n}=u(n \Delta t)$ to $u^{n+1}=u[(n+1) \Delta t]$ namely,

$$
u^{n+1}=u^{n}+\sum_{j=1}^{s} \beta_{j}(\Delta t)^{j} F^{j}(u) .
$$

where $F^{j}=F \circ \ldots \circ F$.

This can be rewritten as

$$
\begin{gathered}
u^{0}=u^{n}, \\
u^{\rho}=u^{n}+\alpha_{\rho} \Delta t F\left(u^{\rho-1}\right),
\end{gathered}
$$

and

$$
u^{n+1}=u^{s},
$$

where $\rho=1,2, \ldots, s$.

For $F(u)$ linear and a six-stage algorithm, we have

$$
u^{n+1}=u^{n}+\sum_{j=1}^{6} \prod_{\rho=7-j}^{6} \alpha_{\rho}(\Delta t)^{j} F^{j}(u),
$$

where $\rho=1,2, \ldots, 6$ and $\prod_{\rho=7-j}^{6} \alpha_{\rho}=\beta_{j}$.

We note that $\beta_{j}=\frac{1}{j !}$ for $j=1,2,3,4$.

The scheme given by (5) is detailed as follows:

$$
u^{0}=u^{n}
$$

$$
\begin{aligned}
& u^{1}=u^{n}+\alpha_{1} \Delta t F\left(u^{0}\right), \\
& u^{2}=u^{n}+\alpha_{2} \Delta t F\left(u^{1}\right), \\
& u^{3}=u^{n}+\alpha_{3} \Delta t F\left(u^{2}\right), \\
& u^{4}=u^{n}+\alpha_{4} \Delta t F\left(u^{3}\right), \\
& u^{5}=u^{n}+\alpha_{5} \Delta t F\left(u^{4}\right), \\
& u^{6}=u^{n}+\alpha_{6} \Delta t F\left(u^{5}\right),
\end{aligned}
$$

and

$$
u^{n+1}=u^{6}
$$

Thus, we have the following relationships

$$
\begin{aligned}
& \alpha_{1}=\frac{\beta_{6}}{\beta_{5}}, \\
& \alpha_{2}=\frac{\beta_{5}}{\beta_{4}} .
\end{aligned}
$$

We consider the usual linear test equation, $u_{t}=\lambda u$ where $\lambda=x+y I$. The exact solution to this test equation can be easily obtained as

$$
u(t+\Delta t)=\exp (\Delta t(x+y I)) u(t),
$$

where $\Delta t$ is the time step.

Using the notation of Albrecht [1], the Runge Kutta solution [19] has the following form:

$u_{n+1}=\left(1+(\lambda k) b^{T} e+(\lambda k)^{2} b^{T} A e+\ldots+(\lambda k)^{s} b^{T} A^{s-1} e\right) u_{n}$,

where $k=\Delta t$ and $e=(1,1,1, \ldots, 1) \in \mathfrak{R}^{s}$.

If $\beta_{j}=b^{T} A^{j-1} e$, then we can rewrite (3) as [19]

$$
u_{n+1}=\left(1+(\lambda k) \beta_{1}+\ldots+(\lambda k)^{s} \beta_{s}\right) u_{n},
$$

or

$$
u_{n+1}=\left(P_{s}+I F_{s}\right) u_{n},
$$

where,

$$
\begin{array}{r}
P_{s}=1+k x \beta_{1}+k^{2}\left(x^{2}-y^{2}\right) \beta_{2}+k^{3}\left(x^{3}-3 x y^{2}\right) \beta_{3}+ \\
k^{4}\left(x^{4}-6 x^{2} y^{2}+y^{4}\right) \beta_{4}+\ldots
\end{array}
$$

and

$$
\begin{gathered}
F_{s}=k y \beta_{1}+k^{2}(2 x y) \beta_{2}+k^{3}\left(3 x^{2} y-y^{3}\right) \beta_{3}+ \\
k^{4}\left(4 x^{3} y-4 x y^{3}\right) \beta_{4}+k^{5}\left(5 x^{4} y-10 x^{2} y^{3}+y^{5}\right) \beta_{5}+\ldots
\end{gathered}
$$


We next consider the following definition:

Definition 1 (Van Der Howen and Sommeijer [26])

The Runge Kutta scheme given by (16) is dissipative of order $p$ if

$$
\exp (x k)-\left|P_{s}+I F_{s}\right|=O\left(k^{p+1}\right),
$$

and dispersive of order $q$ if

$$
h y-\tan ^{-1}\left(\frac{F_{s}}{P_{S}}\right)=O\left(k^{q+1}\right) .
$$

In [26], Van Der Houven and Sommeijer consider the case when $x=0$ (exact solution is non-dissipative). For a fourth-order six-stage method with $x=0, P_{6}$ and $F_{6}$ can be written as

$$
\begin{gathered}
P_{6}=1-\beta_{2}(y k)^{2}+\beta_{4}(y k)^{4}-\beta_{6}(y k)^{6}, \\
F_{6}=y k-\beta_{3}(y k)^{3}+\beta_{5}(y k)^{5},
\end{gathered}
$$

with $\beta_{2}=\frac{1}{2}, \beta_{3}=\frac{1}{6}$ and $\beta_{4}=\frac{1}{24}$. as

The dissipation and dispersion errors can be obtained

$$
E_{A}(y k)=1-\left|P_{6}+I F_{6}\right|
$$

and

$$
E_{\phi}(y k)=y k-\tan ^{-1}\left(\frac{F_{6}}{P_{6}}\right) .
$$

We now describe how Tselios and Simos [25] have obtained their optimal parameters for $\beta_{5}$ and $\beta_{6}$ respectively.

\subsection{Approach by Tselios and Simos [25]}

If we expand (24) and (25) using Taylor series, we obtain an estimate of the dissipation and dispersion errors as [25]

$$
\begin{array}{r}
E_{A}(y k)=(y k)^{6}\left(\frac{1}{144}-\beta_{5}+\beta_{6}\right)+ \\
(y k)^{8}\left(-\frac{1}{1152}+\frac{\beta_{5}}{6}-\frac{\beta_{6}}{2}\right)+(y k)^{10}\left(-\frac{\left(\beta_{5}\right)^{2}}{2}+\frac{\beta_{6}}{24}\right) \\
+(y k)^{12}\left(\frac{1}{41472}+\frac{\left(\beta_{5}\right)^{2}}{2}+\right. \\
\left.\beta_{5}\left(-\frac{1}{144}-\beta_{6}\right)+\frac{\beta_{6}}{144}\right),
\end{array}
$$

and

$$
\begin{array}{r}
E_{\phi}(y k)=(y k)^{5}\left(\frac{1}{120}-\beta_{5}\right)+(y k)^{7}\left(-\frac{1}{336}+\frac{\beta_{5}}{2}-\beta_{6}\right) \\
+(y k)^{9}\left(-\frac{1}{5184}-\frac{\beta_{5}}{24}+\frac{\beta_{6}}{6}\right) \\
+(y k)^{11}\left(\frac{1}{19008}+\left(\beta_{5}\right)^{2}+\beta_{5}\left(-\frac{1}{72}-\beta_{6}\right)\right),
\end{array}
$$

where $E_{A}$ and $E_{\phi}$ are the dissipation and dispersion errors respectively.

Estimates of the magnitude of the dissipation and dispersion errors have been obtained by Tselios and Simos [25] as follows:

$$
\begin{aligned}
& \left|E_{A}\left(\beta_{5}, \beta_{6}\right)\right|=\left(\left(\frac{1}{144}-\beta_{5}+\beta_{6}\right)^{2}\right. \\
& +\left(-\frac{1}{1152}+\frac{\beta_{5}}{6}-\frac{\beta_{6}}{2}\right)^{2} \\
& +\left(-\frac{\left(\beta_{5}\right)^{2}}{2}+\frac{\beta_{6}}{24}\right)^{2}+ \\
& \left.\left(\frac{1}{41472}+\frac{\left(\beta_{5}\right)^{2}}{2}+\beta_{5}\left(-\frac{1}{144}-\beta_{6}\right)+\frac{\beta_{6}}{144}\right)^{2}\right)^{1 / 2} \\
& \text { and } \\
& \begin{array}{r}
\left|E_{\phi}\left(\beta_{5}, \beta_{6}\right)\right|=\left(\left(\frac{1}{120}-\beta_{5}\right)^{2}+\left(-\frac{1}{336}+\frac{\beta_{5}}{2}-\beta_{6}\right)^{2}\right. \\
+\left(\frac{1}{5184}-\frac{\beta_{5}}{24}+\frac{\beta_{6}}{6}\right)^{2}+ \\
\left.\left(\frac{1}{19008}+\left(\beta_{5}\right)^{2}+\beta_{5}\left(-\frac{1}{72}-\beta_{6}\right)\right)^{2}\right)^{1 / 2}
\end{array}
\end{aligned}
$$

We note that Tselios and Simos [25] minimize the quantity, $\left(E_{A}\left(\beta_{5}, \beta_{6}\right)\right)^{2}+\left(E_{\phi}\left(\beta_{5}, \beta_{6}\right)\right)^{2} \quad$ using the Levenberg Marquart method [20] and obtain

$$
\beta_{5}=0.008267383750863793
$$

and

$$
\beta_{6}=0.00121166825454822479 \text {. }
$$

The coefficients proposed by Mead and Renaut [19] are $\beta_{5}=0.00556$ and $\beta_{6}=0.00093$.

Hu et al. [14] found that the optimal values of $\beta_{5}$ and $\beta_{6}$ are 0.00781005 and 0.0013214 respectively. They minimize the following integral,

$$
\int_{0}^{\pi / 2}\left|r-r_{e}\right|^{2} d(y k)
$$

where $\quad r=\frac{u^{n+1}}{u^{n}}=1+z \beta_{1}+\ldots+z^{s} \quad \beta_{s}$ and $r_{e}=\frac{u(t+k)}{u(t)}=\exp (k(x+y I))$.

In the following section, we describe some of our techniques of optimisation $[2,4]$ and in section 5 , we use these techniques to compute optimal values of $\beta_{5}$ and $\beta_{6}$. 


\section{Minimized Integrated Exponential Error for Low Dispersion and Low Dissipation}

In this section, we describe briefly the technique of Minimized Integrated Exponential Error for Low Dispersion and Low Dissipation (MIEELDLD). This technique have been introduced in Appadu and Dauhoo [2], Appadu and Dauhoo [3]. We now give a resume of how we have derived this technique of optimisation.

Suppose the amplification factor of the numerical scheme when applied to the 1-D linear advection equation, given by

$$
\frac{\partial u}{\partial t}+\beta \frac{\partial u}{\partial x}=0
$$

is

$$
\xi=A+I B .
$$

Then the modulus of the Amplification Factor, $(A F M)$ and the relative phase error, $(R P E)$ are calculated as

$$
A F M=|\xi|,
$$

and

$$
R P E=-\frac{1}{c w} \tan ^{-1} \frac{B}{A},
$$

where $c$ and $w$ are the CFL number and phase angle respectively.

For a scheme to have Low Dispersion and Low Dissipation, we require

$$
|1-R P E|+(1-A F M) \longrightarrow 0 .
$$

The quantity, $|1-R P E|$ measures dispersion error while $(1-A F M)$ measures dissipation error. Also when dissipation neutralises dispersion optimally, we have,

$$
|| 1-R P E|-(1-A F M)| \rightarrow 0 \text {. }
$$

Thus on combining these two conditions, we get the following condition necessary for dissipation to neutralise dispersion and for low dispersion and low dissipation character to be satisfied:

$$
\begin{array}{r}
\text { eldld }=|| 1-R P E|-(1-A F M)|+ \\
(|1-R P E|+(1-A F M)) \longrightarrow 0 .
\end{array}
$$

Similarly, we expect

$$
\begin{array}{r}
\text { eeldld }=\exp (|| 1-R P E|-(1-A F M)|)+ \\
\exp (|1-R P E|+(1-A F M))-2 \longrightarrow 0
\end{array}
$$

in order for Low Dispersion and Low Dissipation properties to be achieved.

The measure, eeldld denote the exponential error for low dispersion and low dissipation. The reasons why we prefer eeldld over eldld is because the former is generally more sensitive to perturbations [2].

We next explain how the integration process is performed in order to obtain the optimal parameter(s).

\section{Only one parameter involved}

If the CFL is the only parameter, we compute

$$
\int_{0}^{w_{1}} \text { eeldld } d w
$$

for a range of $w \in\left[0, w_{1}\right]$, and this integral will be a function of $c$. The optimal CFL is the one at which the integral quantity is closest to zero.

\section{Two parameters are involved}

We next consider a case where two parameters are involved and whereby we would like to optimise these two parameters.

Suppose we want to obtain an improved version of the Fromm's scheme which is made up of a linear combination of Lax-Wendroff (LW) and Beam-Warming (BW) schemes. Suppose we apply BW and LW in the ratio $\lambda: 1-\lambda$. This can be done in two ways.

In the first case, if we wish to obtain the optimal value of $\lambda$ at any CFL, then we compute

$$
\int_{0}^{c_{1}} \int_{0}^{w_{1}} \text { eeldld } d w d c
$$

which will be in terms of $\lambda$.

The value of $c_{1}$ is chosen to suit the region of stability of the numerical scheme under consideration while $w_{1} \in[0,1.1]$.

The second way to optimise a scheme made up of a linear combination of Beam-Warming and Lax-Wendroff is to compute the IEELDLD as $\int_{0}^{w_{1}}$ eeldld $d w$ and the integral obtained in that case will be a function of $c$ and $\lambda$. Then a 3-D plot of this integral with respect to $c \in\left[0, c_{1}\right]$ and $\lambda \in[0,1]$ enables the respective optimal values of $c$ and $\lambda$ to be located. The optimised scheme obtained will be defined in terms of both a CFL and the optimal value of $\lambda$ to be used.

Considerable and extensive work on the technique of Minimised Integrated Exponential Error for Low Dispersion and Low Dissipation has been carried out in Appadu and Dauhoo [2], Appadu and Dauhoo [3], Appadu $[4,5,6]$.

In Appadu and Dauhoo [2], we have obtained the optimal CFL for some explicit methods like Lax-Wendroff, Beam-Warming, Crowley, Upwind Leap-Frog and Fromm's schemes. In Appadu and Dauhoo [3], we have combined some spatial discretisation schemes with the optimised time discretisation method proposed by Tam and Webb [23] in order to approximate the 1-D linear advection equation. These spatial derivatives are: a 
standard 7-point and $6^{\text {th }}$-order central difference scheme (ST7), a standard 9-point and $8^{\text {th }}$-order central difference scheme (ST9) and optimised spatial schemes designed by Tam and Webb [23], Lockard et al. [17], Zingg et al. [28], Zhuang and Chen [29] and Bogey and Bailly [10]. The results from some numerical experiments were quantified into dispersion and dissipation errors and we have found that the quality of the results is dependent on the choice of the CFL number even for optimised methods, though to a much lesser degree as compared to standard methods. Moreover, in Appadu [2], we have obtained the optimal CFL of some multi-level schemes in 1-D. These schemes are high order in space and time and have been designed by Wang and Liu [27]. We have also optimised the parameters in the family of Third Order schemes proposed by Takacs [22]. The optimal CFL of the 2-D CFLF4 scheme which is a composite method made up of Corrected Lax-Friedrichs and the two-step Lax-Friedrichs developed by Liska and Wendroff [16] has been computed and some numerical experiments have been performed such as: 2-D solid body rotation test [18], 2-D acoustics [15] and 2-D circular riemann problem [18]. We have shown that better results are obtained when the optimal parameters obtained using MIEELDLD are used.

The technique of MIEELDLD has been extended from Computational Fluid Dynamics framework to Computational Aeroacoustics and used to construct high-order methods [7] which approximate the 1-D linear advection equation. Modifications to the spatial discretization schemes designed by Tam and Webb [23], Lockard et al. [17], Zingg et al. [28] and Bogey and Bailly [10] have been obtained, and also a modification to the temporal scheme developed by Tam et al. [24] has been obtained. These novel methods obtained using MIEELDLD have in general better dispersive properties as compared to the existing optimised methods [7].

Since, in this work, we have estimates for the dissipation and dispersion errors, we do not work with the quantities, $|1-R P E|$ and $(1-A F M)$. Expressions for the dissipation and dispersion errors of the six-stage fourth-order Runge-Kutta scheme are given by (26) and (27) respectively where $\beta_{5}$ and $\beta_{6}$ are the two parameters. Hence, the measure, eldld given by (32) is equivalent to

$$
M_{1}=|| E_{\phi}|-| E_{A}||+\left(\left|E_{\phi}\right|+\left|E_{A}\right|\right) .
$$

For dissipation to neutralise dispersion and for low dispersion and low dissipation properties to be satisfied, we require

$$
M_{1}=|| E_{\phi}|-| E_{A}||+\left(\left|E_{\phi}\right|+\left|E_{A}\right|\right) \longrightarrow 0 .
$$

From (36), we can deduce that

$$
M_{2}=\left(\left|E_{\phi}\right|-\left|E_{A}\right|\right)^{2}+\left(\left|E_{\phi}\right|+\left|E_{A}\right|\right)^{2} \longrightarrow 0
$$

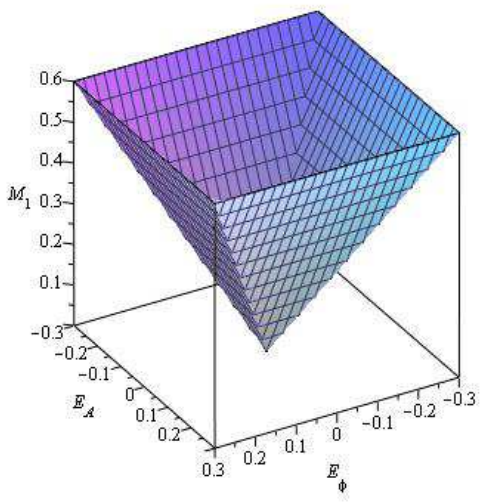

Fig. 1: Plot of the metric, $M_{1}$ vs $E_{\phi}$ vs $E_{A}$

The measure, eeldld given by (33) is equivalent to

$$
M_{3}=\exp || E_{\phi}|-| E_{A}||+\exp \left(\left|E_{\phi}\right|+\left|E_{A}\right|\right)-2 \longrightarrow 0 .
$$

From (38), we can deduce that

$$
M_{4}=\exp \left(\left|E_{\phi}\right|-\left|E_{A}\right|\right)^{2}+\exp \left(\left|E_{\phi}\right|+\left|E_{A}\right|\right)^{2}-2 \longrightarrow 0 .
$$

We note that (36), (37), (38), (39) must be satisfied if we require dissipation to neutralise dispersion and also for low dispersion and low dissipation character to be satisfied.

We next obtain 3D plots of the four measures, all vs dispersion error vs dissipation error in Figs. (1, 2, 3, 4). Dispersion error can be negative if there is phase lag. It can be seen that the measure is zero only when dispersion error and dissipation error are both equal to zero for all the four measures.

The reason why we derive $M_{2}$ and $M_{4}$ is because we use a nonlinear programme (NLP) to compute the minimum of a real-valued objective function. Most of the algorithms used by NLP assume that the objective function and constraints are twice continuously differentiable. However, NLP will sometimes succeed even if these conditions are not met.

\section{Finding optimal values of the two parameters in Runge-Kutta schemes}

We now consider the dispersion and dissipation errors given by (26) and (27) respectively. The integrated error from the metric, $M_{1}$ is computed as 


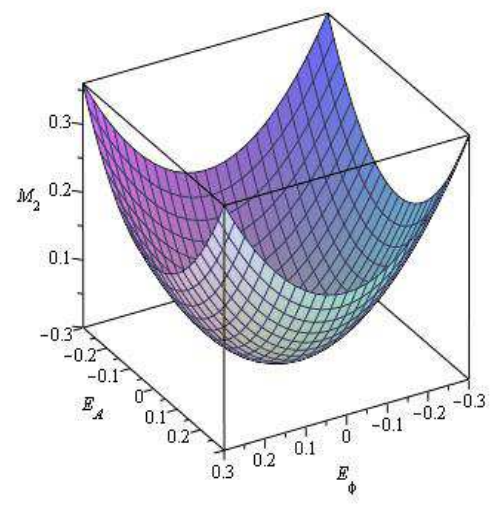

Fig. 2: Plot of the metric, $M_{2}$ vs $E_{\phi}$ vs $E_{A}$

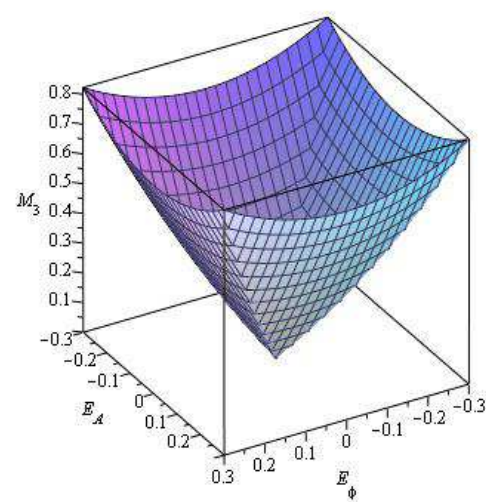

Fig. 3: Plot of the metric, $M_{3}$ vs $E_{\phi}$ vs $E_{A}$

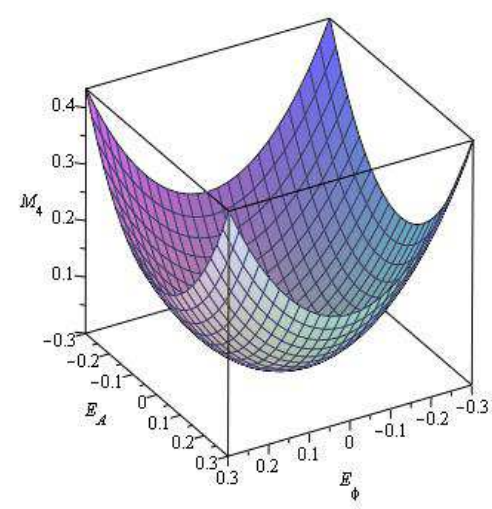

Fig. 4: Plot of the metric, $M_{4}$ vs $E_{\phi}$ vs $E_{A}$

$$
\int_{0}^{1.1}|| E_{\phi}|-| E_{A}||+\left|E_{\phi}\right|+\left|E_{A}\right| d(y k) .
$$

A 3-D plot and contour plot of this integrated error vs $\beta_{5} \in[0,0.02]$ vs $\beta_{6} \in[0,0.0015]$ is shown in Fig. (5) and we can deduce that the approximate values for $\beta_{5}$ and $\beta_{6}$ are 0.008 and 0.00132 respectively.

The Integrated Error for the metric, $M_{2}$ is computed as

$$
\int_{0}^{1.1}\left(\left|E_{\phi}\right|-\left|E_{A}\right|\right)^{2}+\left(\left|E_{\phi}\right|+\left|E_{A}\right|\right)^{2} d(y k),
$$

and a plot of this integrated error vs $\beta_{5}$ vs $\beta_{6}$ is shown in Fig. (6). We then use optimisation function to obtain the optimal values of $\beta_{5}$ and $\beta_{6}$ as $0.00813684557939976447 \quad$ and 0.00136374541073363914 respectively.

A plot of the Integrated Error from $M_{3}$ vs $\beta_{5}$ vs $\beta_{6}$ is shown in Fig. (7). However, we cannot locate the optimal values of $\beta_{5}$ and $\beta_{6}$ accurately. It is for this reason precisely that we define the metric, $M_{4}$.

A plot of the integrated error from $M_{4}$ vs $\beta_{5}$ vs $\beta_{6}$ is shown in Fig. (8) and we obtain the optimal values of $\beta_{5}$ and $\beta_{6}$ as 0.00813684556860650062 and 0.00136374541912936944 .

We observe that the optimal values of $\beta_{5}$ and $\beta_{6}$ obtained using the metrics, $M_{2}$ and $M_{4}$ agree to 10 decimal places. Hence, the values of $\beta_{5}$ and $\beta_{6}$ that we use for our new scheme are 0.0081368456 and 0.0013637454 respectively.

\subsection{Comparison of known methods with new method}

In our work, we shall take $\beta_{5}=0.0081368456$ and $\beta_{6}=0.0013637454$. Table (1) gives values for $\beta_{5}$ and $\beta_{6}$ for four temporal Runge-Kutta schemes namely; Hu et al., Mead and Renaut, Tselios and Simos and our new method. We use Eqs. (26) and (27) to obtain the plots of the dissipation and dispersion errors vs $y k$.

Figs. (9) and (10) shows the plot of the magnitude of the dispersion and dissipation errors vs $y k$ for four temporal Runge-Kutta schemes.

With increase in $y k$, the magnitude of the dispersion error increases as expected. The scheme proposed by Mead and Renaut and $\mathrm{Hu}$ et al. have significantly more dispersion error than the other two schemes. The dispersion error from Tselios and Simos [25] is slightly smaller than the one by Appadu for $y k \in[0,0.8]$ but for $y k>0.8$, the dispersion error from Appadu is much less than the other three schemes. 

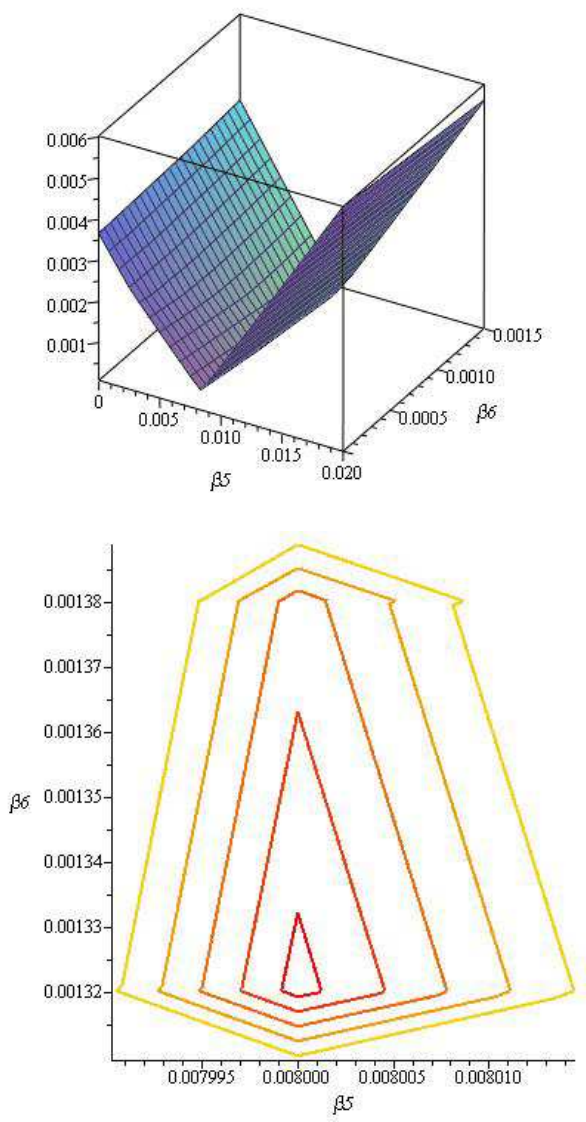

Fig. 5: Plot of Integrated Error from $M_{1}$ vs vs $\beta_{5}$ vs $\beta_{6}$.

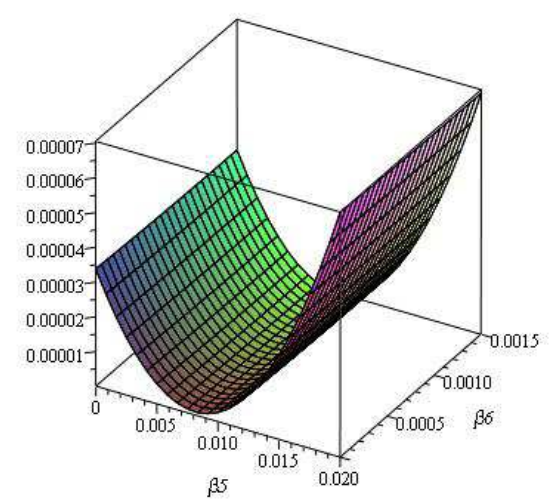

Fig. 6: Plot of Integrated Error from $M_{2}$ vs vs $\beta_{5}$ vs $\beta_{6}$.

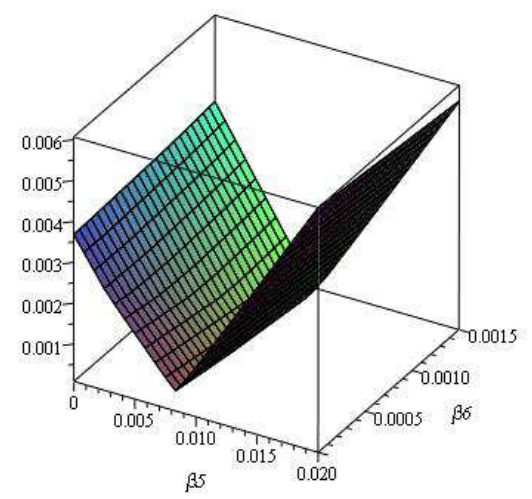

Fig. 7: Plot of Integrated Error from $M_{3}$ vs vs $\beta_{5}$ vs $\beta_{6}$.

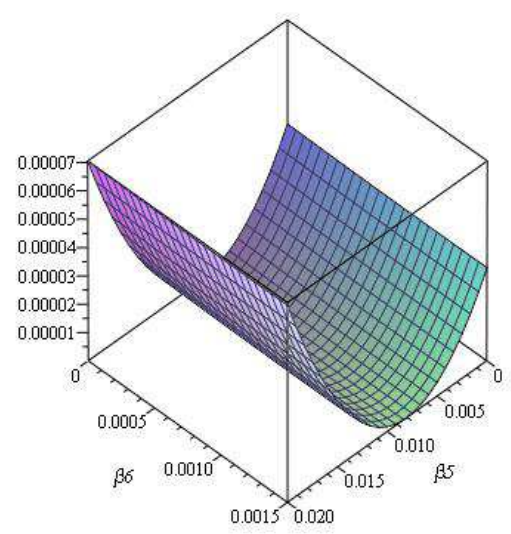

Fig. 8: Plot of Integrated Error from $M_{4}$ vs vs $\beta_{5}$ vs $\beta_{6}$.

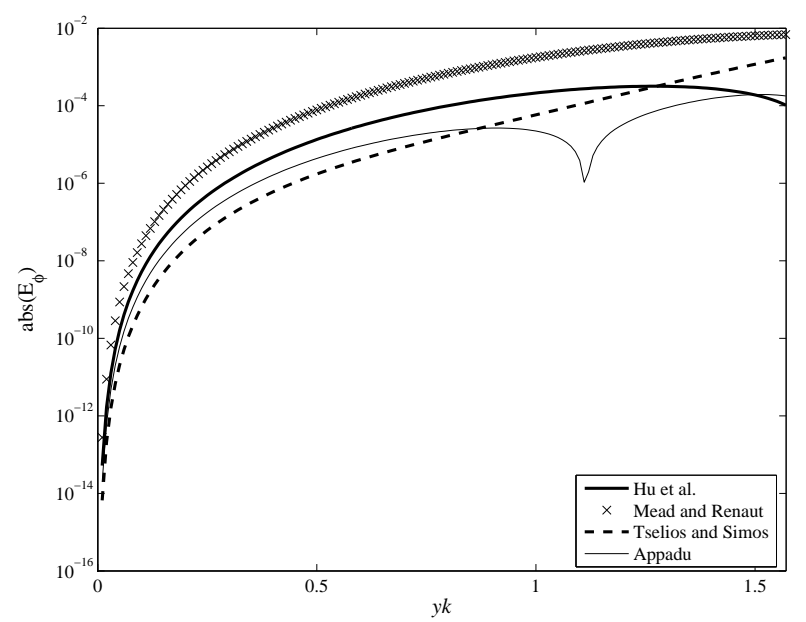

Fig. 9: Plot of the magnitude of the dispersion error on a logarithmic scale vs $y k \in[0,1.5]$ for some temporal discretisation schemes. 
Table 1: Comparing the coefficients of $\beta_{5}$ and $\beta_{6}$ for the four Runge Kutta schemes

\begin{tabular}{lll}
\hline Method & $\beta_{5}$ & $\beta_{6}$ \\
\hline Hu et al. [14] & 0.00781005 & 0.00132141 \\
\hline Mead and Renaut [19] & 0.00556 & 0.00093 \\
\hline Tselios and Simos [25] & 0.0082673838 & 0.0012116682 \\
\hline Appadu & 0.0081368456 & 0.0013637454 \\
\hline
\end{tabular}

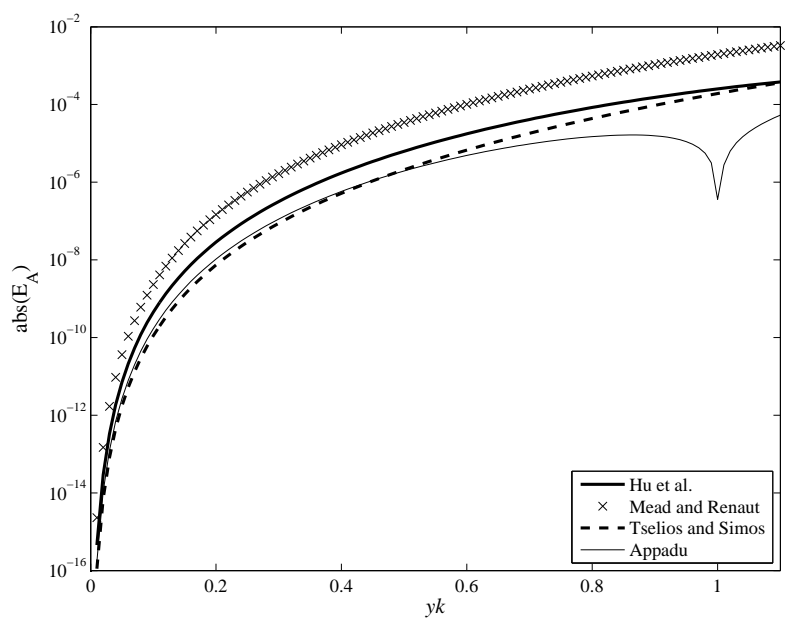

Fig. 10: Plot of the magnitude of the dissipation error on a logarithmic scale vs $y k \in[0,1.1]$ for some temporal discretisation schemes.

The scheme proposed by Mead and Renaut [19] has the largest dissipation error followed by the one proposed by $\mathrm{Hu}$ et al. [14]. The scheme by Tselios and Simos, Appadu have almost the same dissipation error for $y k \in[0,0.5]$ but for $y k>0.5$, the scheme by Appadu has significantly less dissipation error.

\section{Numerical Experiments}

\subsection{Convective Wave Equation}

We consider the following partial differential equation:

$$
\frac{\partial u}{\partial t}+\frac{\partial u}{\partial x}=0
$$

At $t=0$, we have a Gaussian profile, $u_{0}=0.5 \exp \left[-\left(\frac{x}{3}\right)^{2}\right]$ [25] and the domain extends from $x_{\min }=-50$ to $x_{\max }=t+50$. The analytical solution is $u(x, t)=0.5 \exp \left[-\left(\frac{x-t}{3}\right)^{2}\right]$.
A nine point centred difference scheme of eight order is used for the spatial discretization which is given by

$$
\begin{aligned}
\frac{\partial u}{\partial x} & \approx \frac{1}{h}\left(-\frac{4}{5}\left(u_{i-1}^{n}-u_{i+1}^{n}\right)+\frac{1}{5}\left(u_{i-2}^{n}-u_{i+2}^{n}\right)\right. \\
& \left.-\frac{4}{105}\left(u_{i-3}^{n}-u_{i+3}^{n}\right)+\frac{1}{280}\left(u_{i-4}^{n}-u_{i+4}^{n}\right)\right) .
\end{aligned}
$$

Our new method is compared with

(i) the fourth-order six-stage Runge-Kutta scheme of $\mathrm{Hu}$ et al [14].

(ii) the fourth-order six-stage Runge-Kutta scheme of Mead and Renaut [19].

(iii) the fourth-order six-stage Runge-kutta scheme of Tselios and Simos [25]

In all four cases, we have used a nine point centred difference scheme of eight order for the spatial discretization scheme.

Our aim is to obtain values of the error rate with respect to the $L_{1}$-norm and the Total Mean Square Error [22] which are computed as

$$
E_{\text {num }}=h \sum_{i=1}^{N}\left|u_{c}-u_{e}\right|,
$$

and

$$
\frac{1}{N} \sum_{i=1}^{N}\left(u_{c}-u_{e}\right)^{2},
$$

where $u_{c}$ and $u_{e}$ are the computed and exact values, respectively and $N$ is the number of spatial grid points. The Total Mean Square Error is equivalent to the sum of the dispersion and dissipation errors as shown by Takacs [22]. We then plot these two types of errors vs the CFL number and find out how these two errors vary with the CFL number. We consider two cases namely, $t=400$ and $t=1000$.

\section{Case 1}

We consider the case when time, $t=400$, and spatial step, $h=0.5$. Since, $\mathrm{CFL}=k / h$, and $k=400 / n_{\max }$, then

$$
C F L=800 / n_{\max },
$$

where $n_{\text {max }}$ is the number of time steps.

We use some CFL numbers to satisfy (46) such that $n_{\max }$ is an integer. Once $n_{\max }$ has been determined, we can find the time step, $k$. The CFL numbers we consider and the corresponding values of $n_{\max }$ and $k$ are shown in Table (2).

Fig. (11) shows the variation of the error rate vs CFL for four numerical schemes. The general tendency is that the error is not much affected by the choice of the CFL number except in the case of Mead and Renaut. We have 
Table 2: Some values of CFL, $n_{\max }$ and $k$ for time, $t=400$ and spatial step, $h=0.5$

\begin{tabular}{lll}
\hline CFL & $n_{\max }$ & $k$ \\
\hline 0.1 & 80000 & 0.05 \\
\hline 0.2 & 4000 & 0.1 \\
\hline 0.4 & 2000 & 0.2 \\
\hline 0.64 & 1250 & 0.32 \\
\hline 0.80 & 1000 & 0.40 \\
\hline
\end{tabular}

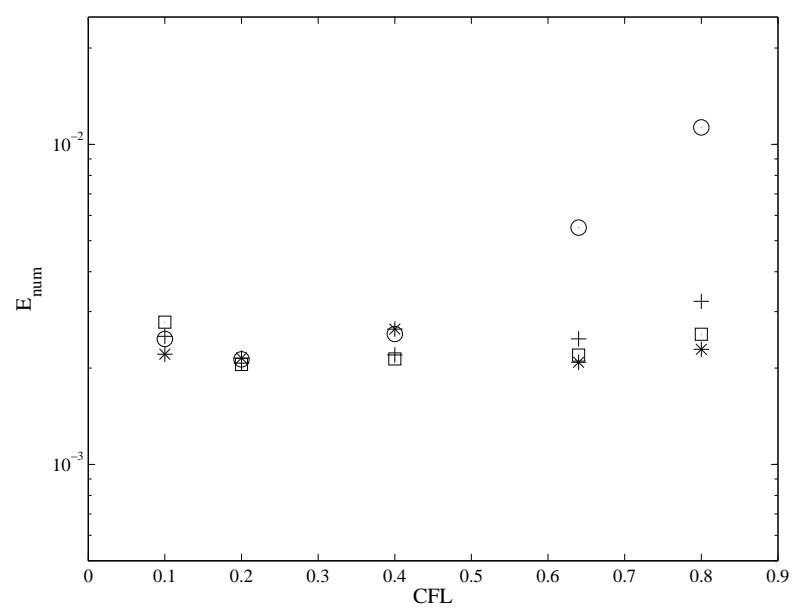

Fig. 11: Error rate as a function of CFL, using Runge-Kutta scheme of Hu et al ' $+^{\prime}$, Mead and Renaut 'o', Tselios and Simos $' \square$ ' and our new scheme, New ${ }^{\prime} *^{\prime}$, all combined with a ninepoint central difference scheme of eight order at time, $t=400$ and spatial step, $h=0.5$.

considered five CFL numbers namely $0.1,0.2,0.4,0.64$ and 0.8. At CFL 0.1, 0.64 and 0.8, our scheme is the most effective one. At CFL 0.4, the schemes by Tselios and Simos and $\mathrm{Hu}$ et al. are the most effective.

Fig. (12) shows the variation of the Total Mean Square Error vs the CFL number. The general tendency is that the error increases with increase in CFL number for the scheme by Mead and Renaut and $\mathrm{Hu}$ et al. Our scheme followed by Tselios and Simos are the most effective ones over the five values of CFL used.

\section{Case 2}

We consider the case when time, $t=1000$ and $h=0.5$. Values of CFL and the corresponding values of $n_{\max }$ and $k$ are shown in Table (3). The difference is since the time has now been multiplied by 2.5 , therefore the values of $n_{\max }$ are multiplied by 2.5 , as compared to the values in Table (2).

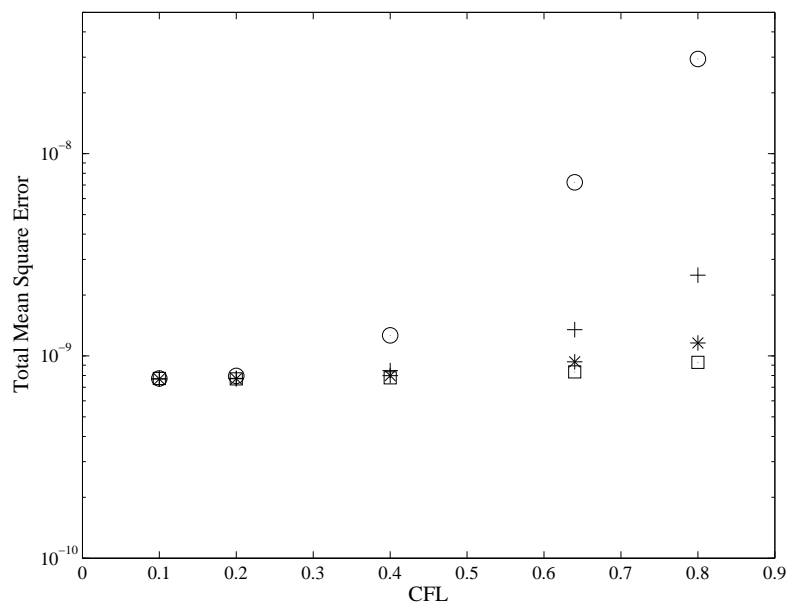

Fig. 12: Total Mean Square Error as a function of CFL, using Runge-Kutta scheme of $\mathrm{Hu}$ et al ' + ', Mead and Renaut 'o', Tselios and Simos ' $\square$ ' and our new scheme, New ' $*$ ', all combined with a nine-point central difference scheme of eight order at time, $t=400$ and spatial step, $h=0.5$.

Table 3: Some values of CFL, $n_{\max }$ and $k$ for time, $t=1000$ and spatial step, $h=0.5$

\begin{tabular}{lll}
\hline CFL & $n_{\max }$ & $k$ \\
\hline 0.1 & 20000 & 0.05 \\
\hline 0.2 & 10000 & 0.1 \\
\hline 0.4 & 5000 & 0.2 \\
\hline 0.64 & 3125 & 0.32 \\
\hline 0.80 & 2500 & 0.40 \\
\hline
\end{tabular}

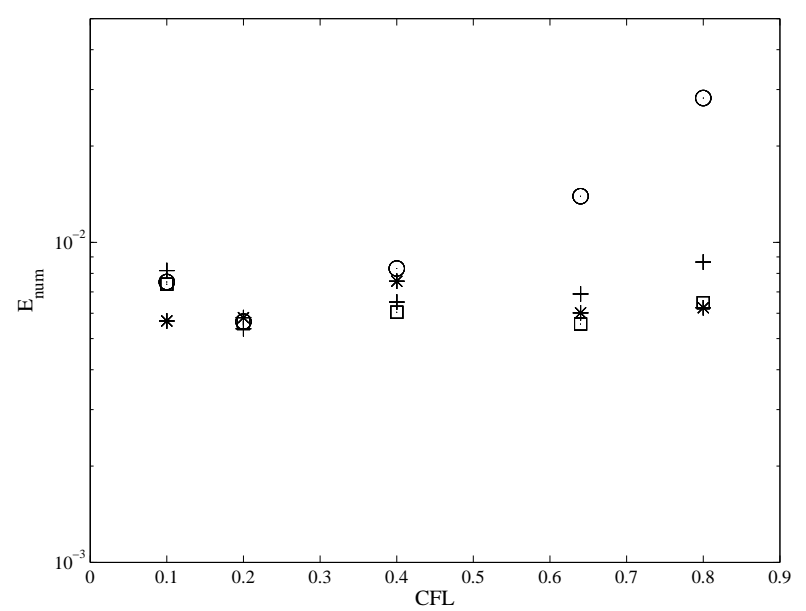

Fig. 13: Error rate as a function of CFL using Runge-Kutta scheme of Hu et al ' $+{ }^{\prime}$, Mead and Renaut ' ${ }^{\prime}$, Tselios and Simos $' \square$ and our new scheme, New ${ }^{\prime} *$, all combined with a nine-point central difference scheme of eight order at time, $t=1000$ and spatial step, $h=0.50$. 


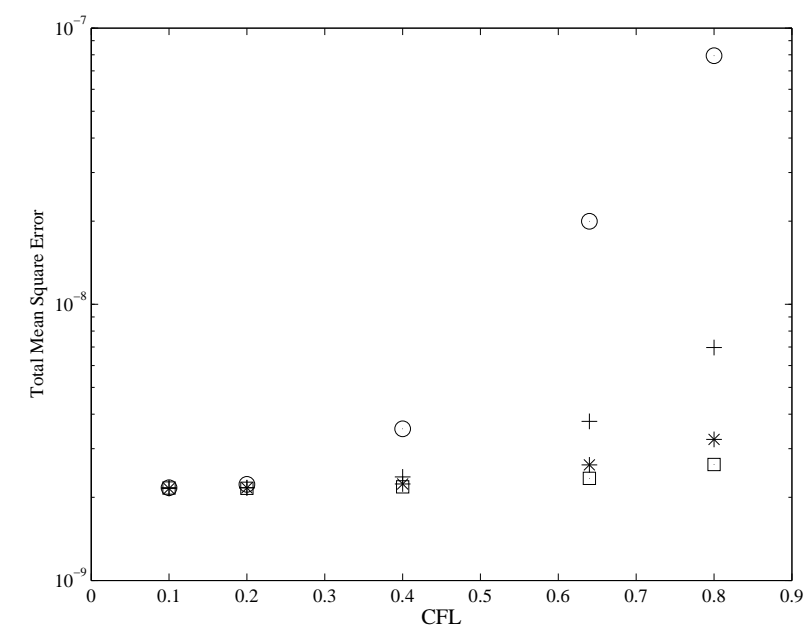

Fig. 14: Total Mean Square Error as a function of CFL using Runge-Kutta scheme of $\mathrm{Hu}$ et al ' + ', Mead and Renaut ' $o$ ', Tselios and Simos ' $\square$ ' and our new scheme, New ' ${ }^{\prime}$, all combined with a nine-point central difference scheme of eight order at time, $t=1000$ and spatial step, $h=0.50$.

Fig. (13) shows the variation of the error rate vs CFL. The values of the error rate are much affected by the CFL number in the case of Runge-Kutta scheme proposed by Mead and Renaut. At CFL 0.1, 0.64 and 0.8 , our proposed scheme is better. At CFL 0.4 , the scheme by Tselios and Simos is slightly better. Over the five CFL numbers, our scheme is generally the best one followed by the scheme of Tselios and Simos.

Fig. (14) shows the variation of the Total Mean Square Error vs CFL. The values of the Total Mean Square Error are much affected by CFL in the case of Runge Kutta schemes by $\mathrm{Hu}$ et al., Mead and Renaut.

\subsection{Spherical Wave Problem [12]}

We consider the following problem

$$
\frac{\partial u}{\partial t}+\frac{\partial u}{\partial r}+\frac{u}{r}=0
$$

where $5 \leq r \leq 315$ and $t>0$.

The initial condition is $u(r, 0)=0$ for $5 \leq r \leq 315$.

The boundary condition is $u(5, t)=\sin (\pi t / 3)$ for $0<t<300$.

The analytic solution is given by

$u(r, t)=0$ for $r>t+5$ and $u(r, t)=\frac{5}{r} \sin (\pi(t-r+5) / 3)$ for $r \leq t+5$.

Since we have found from section 6.1 that low CFL numbers are preferred to minimise both dispersion and dissipation errors, we shall use a small CFL for this experiment, say 0.1. We perform the numerical experiment at time, $t=300$ at $\mathrm{CFL}=0.1$ with time step 0.02 and spatial step 0.2 . The error rate with respect to $L_{1}$ error and the Total Mean Square Error are compared in Table (4). The results are shown in Fig. (15). Once again, it is seen that the scheme by Appadu is the best one.
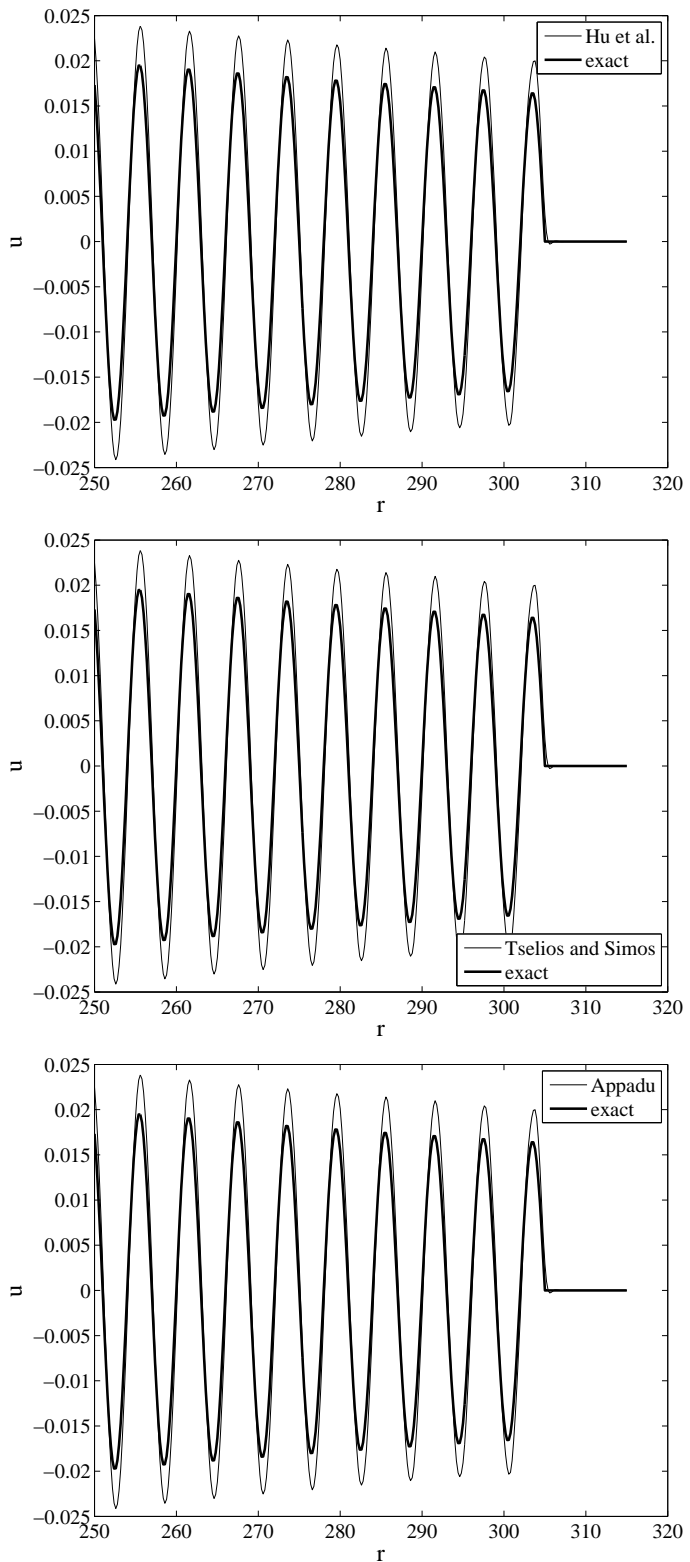

Fig. 15: Solution of the spherical wave problem with three temporal Runge-Kutta schemes, all coupled with with a ninepoint centred difference scheme of eight order at time, $t=300$ with spatial step 0.2 and time step 0.02 . 
Table 4: Spherical wave problem with spatial step 0.2 and time step 0.02

\begin{tabular}{lll}
\hline Method & Error rate $-L_{1}$ error & Total Mean Square Error \\
\hline Hu et al. & 3.081115 & $3.863205 \times 10^{-4}$ \\
\hline Tselios and Simos & 3.081116 & $3.863204 \times 10^{-4}$ \\
\hline Appadu & 3.081114 & $3.863198 \times 10^{-4}$ \\
\hline
\end{tabular}

\section{Conclusion}

We have performed a spectral analysis of the dispersion and dissipation errors of four temporal Runge-Kutta schemes coupled with a nine-point centred difference scheme of eight order and seen that our proposed scheme has the best dispersive and dissipative character. Based on the plots of the error rate of the $L_{1}$ norm and the Total Mean Square Error vs CFL number for the convective wave equation, we conclude that our proposed scheme is better than the other three schemes when $h$ is chosen as 0.50 at two different times, $t=400$ and $t=1000$. Also, our proposed scheme is the best one for the simulation of the spherical wave problem. These demonstrate that our technique of optimisation is effective to construct low dispersion and low dissipation Runge-Kutta schemes. Secondly, we observe that the general trend is that low CFL numbers are more efficient than larger CFL numbers in the case of optimized schemes as opposed to standard schemes.

One possible extension of this work is to take stability into consideration when we optimise the parameters which control the grade and balance of dispersion and dissipation. We will aim to construct low dispersion and low dissipation Runge-Kutta schemes with large range of stability.

\footnotetext{
Nomenclature

$I=\sqrt{ }(-1)$

$k$ : time step

$h$ : spatial step

$n$ : time level

$\beta$ : advection velocity

$c$ : CFL/Courant number

$c=\frac{\beta k}{h}$

$w$ : phase angle in 1-D

$w=\theta h$
}

$R P E$ : relative phase error per unit time step

$A F$ : amplification factor

$A F M=|A F|$

LDLD: Low Dispersion and Low Dissipation

IEELDLD: Integrated Exponential Error for Low Dispersion and Low Dissipation

MIEELDLD: Minimised Integrated Exponential Error for Low Dispersion and Low Dissipation.

\section{Acknowledgement}

This work was funded through the Research Development Programme of the University of Pretoria. The period of funding is from January 2012 to January 2014.

The author wishes to thank the anonymous referees for their valuable comments and suggestions which have helped to improve on the work.

\section{References}

[1] P. Albrecht, The Runge-kutta Theory in a nutshell, SIAM Journal of Numerical Analysis, 33, 1712 (1996).

[2] A. R. Appadu and M. Z. Dauhoo, The Concept of Minimised Integrated Exponential Error for Low Dispersion and Low Dissipation, International Journal for Numerical Methods in Fluids, 65, 578-601 (2011).

[3] A. R. Appadu and M.Z. Dauhoo, An Overview of Some High Order and Multi-Level Numerical Schemes in Computational Aeroacoustics, Proceedings of the World Academy of Science, Engineering and Technology, 38, 365-380 (2009).

[4] A. R.Appadu, Some Applications of the Concept of Minimized Integrated Exponential Error for Low Dispersion and Low Dissipation Schemes, International Journal for Numerical Methods in Fluids, 68, 244-268 (2012).

[5] A. R. Appadu, Comparison of Some Optimisation techniques for Numerical Schemes Discretising Equations with Advection Terms, International Journal of Innovative Computing and Applications, 4, (2012).

[6] A. R. Appadu, Investigating the Shock-Capturing Properties of Some Composite Numerical Schemes for the 1-D Linear Advection Equation, International Journal of Computer Applications in Technology, 43, 79-92 (2012).

[7] A. R.Appadu, The Technique of MIEELDLD in Computational Aeroacoustics, Journal of Applied Mathematics, (2012).

[8] G. Ashcroft and Xin Zhang, Optimized Prefactored Compact Schemes, Journal of Computational Physics, 190, 459-477 (2003).

[9] J. Berland, C. Bogey and C. Bailly, Low-dissipation and lowdispersion fourth-order Runge-Kutta Algorithm, Computers and Fluids, 35, 1459-1463 (2006).

[10] C. Bogey and C. Bailly, A Family of Low Dispersive and Low Dissipative Explicit Schemes for Computing the Aerodynamic Noise, Journal of Computational Physics, 194, 194-214 (2004).

[11] J. Hardin and M.Y. Hussaini, Computational Aeroacoustics, Springer-Verlag, New-York, Berlin, (1992).

[12] J. C. Hardin, J. R. Ristorcelli and C. K. W. Tam (editors), ICASE/LaRC workshop on Benchmark Problems in Computational Aeroacoustics, NASA CP 3300, (1995)

[13] R. Hixon, Evaluation of High-Accuracy MacCormackType Scheme Using Benchmark Problems, NASA Contractor Report 202324, ICOMP-97-03-1997.

[14] F. Q. Hu, M.Y.Hussaini, J.L. Manthey, Low-dissipation and low-dispersion Runge-Kutta schemes for Computational Acoustics, Journal of Computational Physics, 124, 177-191 (1996). 
[15] C. Kim, Multi-dimensional Upwind Leapfrog Schemes and their Applications, Dissertation in partial fulfillment of the requirements for the Degree of Doctor of Philosophy, Aerospace Engineering, University of Michigan. (1997).

[16] R. Liska and B. Wendroff, Composite Schemes for Conservation Laws, SIAM Journal of Numerical Analysis, 35, 2250-2271 (1998).

[17] D. P. Lockard, K. S.Brentner and H. L. Atkins, HighAccuracy Algorithms for Computational Aeroacoustics, AIAA Journal, 33, 246-251 (1995).

[18] Lukacova, Finite Volume Schemes For Multidimensional Hyperbolic Systems Based On The Use Of Bicharacteristics, Applications of Mathematics, 51, 205-228 (2006).

[19] J. L. Mead, R. A. Renaut, Optimal Runge-Kutta methods for first order pseudospectral operators, Journal of Computational Physics, 152, 404-419 (1999).

[20] D. Marquardt, An Algorithm for least-squares estimation of non-linear parameters, SIAM Journal of Applied Mathematics, 11, 431-441 (1993).

[21] D. Stanescu and W. G. Habashi, 2N-Storage Low Dissipation and Dispersion Runge-Kutta schemes for Computational Acoustics, Journal of Computational Physics, 143, 674-681 (1998).

[22] L. Takacs, A Two-Step Scheme for the Advection Equation with Minimized Dissipation and Dispersion errors, Monthly Weather Review, 113, 1050-1065 (1985).

[23] C. K. W. Tam and J. C. Webb, Dispersion-RelationPreserving Finite Difference Schemes for Computational Acoustics, International Journal of Computational Physics, 107, 262-281 (1993).

[24] C. K. W. Tam, J. C. Webb and Z. Dong, A Study of the Short Wave Components in Computational Acoustics, Journal of Computational Acoustics, 1, 1-30 (1993).

[25] K. Tselios and Simos, Runge-Kutta methods with minimal dispersion and dissipation for problems arising from computational acoustics, Journal of Computational and Applied Mathematics, 175, 173-181 (2005).

[26] P. J. Van Der Howen and B. P. Sommeijer, Explicit Ruge-Kutta Nystrom methods with reduced phase errors for computing oscillating oscillations, SIAM Journal on Numerical Analysis, 24, 595-617 (1987).

[27] Wang and Liu, A New Approach to Design HighOrder Schemes, Journal of Computational and Applied Mathematics, 134, 59-67 (2001).

[28] D. W. Zingg, H. Lomax and H. Jurgens, High-Accuracy Finite-Difference Schemes for Linear Wave Propagation, SIAM Journal on scientific Computing, 17, 328-346 (1996).

[29] M. Zhuang and R. F. Chen, Application of HighOrder Optimised Upwind Schemes for Computational Aeroacoustics, AIAA Journal, 40, 443-449 (2002).

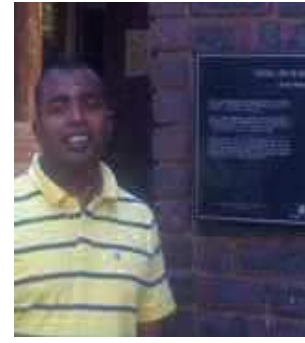

A. R. Appadu is a lecturer in the Department of Mathematics and Applied Mathematics at the University of Pretoria, South Africa. $\mathrm{He}$ received the $\mathrm{PhD}$ degree in Computational Fluid Dynamics at the University of Mauritius. His research interests are in the areas of Computational Fluid Dynamics, Computational Aeroacoustics and Optimisation. He has published research articles in reputed international journals in Applied Mathematics. He is referee of some international journals. 